\title{
Variations
}

Variations

Revue internationale de théorie critique

$8 \mid 2006$

Subjectivités libres et critique de la répression

\section{D'Autres mondes sont possibles et La Planète altermondialiste}

Sascha Goldwasser

\section{(2) OpenEdition}

1 Journals

Édition électronique

URL : http://journals.openedition.org/variations/520

DOI : 10.4000/variations. 520

ISSN : 1968-3960

Éditeur

Les amis de Variations

Édition imprimée

Date de publication : 1 novembre 2006

Pagination : 116-118

Référence électronique

Sascha Goldwasser, "D'Autres mondes sont possibles et La Planète altermondialiste ", Variations [En ligne], 8| 2006, mis en ligne le 27 décembre 2012, consulté le 22 septembre 2020. URL : http:// journals.openedition.org/variations/520 ; DOI : https://doi.org/10.4000/variations.520

Ce document a été généré automatiquement le 22 septembre 2020.

Les ami•e•s de Variations 


\title{
D'Autres mondes sont possibles et La Planète altermondialiste
}

\author{
Sascha Goldwasser
}

\section{RÉFÉRENCE}

Mathieu Colloghan (Dir.), D'autres mondes sont possibles. Retour sur les forums sociaux mondiaux. Seca, Lyon, 2006

Chiara Bonfiglioli et Sebastian Budgen (Dir.), La Planète altermondialiste. Guide critique.

Editions Textuel, coll. La Discorde, Paris, 2006

1 Alors que les études historiques au sujet des Internationales ouvrières abondent, le phénomène des Forums sociaux mondiaux, amorcé en 2001, ne fait pas encore l'objet de recherches universitaires. Deux livres tentent de saisir la pluralité des vues et des courants qui traversent le mouvement contemporain pour un autre monde. Les deux ouvrages collectifs expriment un point de vue politiquement engagé, le premier ouvertement, au nom des Alternatifs (Mathieu Colloghan), le second d'une manière plutôt voilée, en faveur de la LCR (Bonfiglioli/Budgen).

2 Tous deux se heurtent à la difficulté de définir une position théorique cohérente, à partir de laquelle les arguments et motivations des nombreux acteurs de l'altermondialisme puissent être cernés d'une manière critique. L'ouvrage des Alternatifs, D'autres mondes sont possibles, contourne cet obstacle d'une manière assez élégante, en livrant une documentation éclectique des discours, expériences et luttes des personnes parties prenantes du mouvement global. Cette approche correspond manifestement à la vision d'un des principaux initiateurs des Forums, Chico Whitaker, pour qui l'altermondialisme doit formuler et rassembler des propositions particulières, diverses, afin d'éviter d'accoucher d'une synthèse politique appauvrie. L'autre ouvrage, La Planète altermondialiste, évoque l'inspiration théorique et éthique de Whitaker: la théologie de la libération. Michael Löwy en présente les fondateurs spirituels (Leonardo Boff et Frei Betto), au cours d'une des contributions les plus éclairantes. Pareil lien 
entre la théorie et l'engagement n'apparaît que très rarement dans les deux ouvrages, ce qui est plus gênant dans le cas de la contribution trotskiste, qui voudrait analyser les influences intellectuelles de l'altermondialisme.

D'autres mondes sont possibles apporte une pierre plus chatoyante, quoique moins lourde en apparence, en livrant des témoignages authentiques de quelques-unes des figures les plus en vue. On y tombe sur quelques perles et notamment sur de petits essais d'Arundhati Roy, femme de lettres indienne très engagée, dont l'un est intitulé Les dindes aiment-elles Thanksgiving? On trouvera aussi des entretiens avec Raoul Pont (ancien maire de Porto Alegre et représentant de l'aile gauche du Parti des travailleurs du Brésil), avec Luciano Brunet (responsable du budget participatif), ainsi qu'avec Vilson Santin (dirigeant du Mouvement sans terre). Le zapatisme est abordé par Gerardo Fernandez Casanova, du journal mexicain La Jornada, mais le sous-commandant Marcos est absent de la publication. De même, les mobilisations européennes de la dernière période ne font pas partie du panorama. Si proche, si loin? En revanche, la plupart des appels des mouvements sociaux, lancés à l'occasion des Forums mondiaux, sont ici reproduits. Au milieu de ces contributions s'affichent des comptes-rendus et des commentaires, journalistiques, ironiques ou anecdotiques. Par moments, le lecteur se trouve plongé dans l'ambiance euphorique du village global. La Planète altermondialiste vise plus haut, mais tombe à plat. L'ouvrage se veut un guide critique de la pensée des figures de proue intellectuelles, mais il sème la confusion. Pierre Bourdieu se voit affublé du titre de "social-démocrate radical», expression hautement contradictoire et inappropriée, au vu des articles politiques du sociologue. La préface, tout comme la plupart des textes, se réfèrent à un hypothétique clivage idéologique qui opposerait «l'anti-capitalisme » et "l'anti-libéralisme », mais aucun des termes n'est explicité. Il s'agit de coquilles rhétoriques qui ne disposent pas de la force d'un concept. Les auteurs du livre ignoreraient-ils que le libéralisme désigne des courants progressistes dans le monde anglophone?

4 Les coordinateurs nous avertissent que l'unité de la théorie et de la pratique, exigée par Lénine, est impossible, entraînant la "revalorisation du pluralisme » des conceptions. Pourtant, des commentateurs comme Callinicos et Bensaïd défendent un léninisme tout à fait classique dans leurs critiques de Negri et de Holloway, tandis que leur représentation de la prise du pouvoir traverse l'ouvrage comme un fil rouge. Cela apparaît nettement dans les chapitres accordés aux organisateurs et aux responsables politiques servant de référence au sein de l'altermondialisme, et qui occupent une série de chapitres (Bernard Cassen, Hugo Chávez, Susan George, Naomi Klein, Marcos). Comme ces figures politiques n'ont qu'un rapport lointain avec les constructions théoriques en jeu, les passages qui leur sont consacrés ont été confiés à des militants de base de la LCR, qui parfois se laissent aller en parlant du «beau processus de conscientisation des masses ». Retour de Lénine, qui voulait apporter la conscience aux masses depuis l'extérieur, étant donné que leur propre expérience était supposée s'arrêter à un vague syndicalisme apolitique. Cette optique militante ne va pas sans un certain tropisme. Au sujet de Cassen, président d'ATTAC! et co-responsable d'une fraude électorale au sein de son association, l'une des contributions juge: «S'il doit faire face aux accusations d'autoritarisme, il n'est pas désavoué par une base militante qui refuse de se mêler des conflits ». Appréciation erronée, puisque la direction d'ATTAC! a démissionné au cours de cet été; témoignage d'un bel esprit bureaucratique. 
5 Daniel Bensaïd accompagne cette démarche par l'étayage d'un matérialisme historique à l'ancienne qui ne connaît que des révolutions «bourgeoises» ou "prolétariennes », cherchant à faire taire les critiques envers sa propre position grâce à une argumentation philosophique sans failles : « Lénine était le plus avisé, qui distinguait la maladie sénile du socialisme parlementaire de la maladie infantile du communisme. On peut guérir de la seconde, on survit rarement à la première » (p. 130). Le philosophe se montre enclin à faire survivre sa vision infantile de la dictature du prolétariat par le recours au charisme du président Chavez. Au sein du même ouvrage, il se voit judicieusement contredit par Sébastien Ville, soulignant que seul le contre-pouvoir contestataire de la rue était en mesure de neutraliser la tentative de coup d'État à l'encontre de la république bolivarienne, en 2002. Le pouvoir de dire non, voilà un thème cher à Holloway.

6 Au cours de l'ouvrage, on mobilise Lénine contre Negri, Engels contre Foucault, on oppose Cassen, Chevènement et Castro à Holloway, on loue le keynésianisme étatique de Stiglitz. Manifestement, la critique du pouvoir, de l'État et de l'électoralisme fait peur. Vers la fin du livre, le lecteur découvre le visage souriant de l'altermondialisme, celui du féminisme et de la "dissidence démocratique ", portés par Arundhati Roy et Aminata Traoré ; l'Inde et l'Afrique volent à notre secours. Il serait préférable de donner la parole aux théoriciens altermondialistes eux-mêmes à la prochaine occasion, bien que l'ouvrage contienne trois chapitres intéressants sur une vingtaine au total.

\section{AUTEURS}

\section{SASCHA GOLDWASSER}

Doctorant en philosophie à l'Université de Strasbourg, militant d'ATTAC! 